\title{
Formación de titanato de aluminio por reacción en estado sólido de alumina y titania
}

\author{
R. URIBE', C. BAUDÍN \\ Instituto de Cerámica y Vidrio (CSIC). 28500,Arganda del Rey, Madrid, España. \\ ${ }^{1}$ En estancia procedente del Instituto Universitario de Tecnología Dr. "F.R.P." Caracas 1040, Venezuela.
}

\begin{abstract}
En el presente trabajo se ha estudiado el efecto que tienen las condiciones de tratamiento térmico (entre 1350 y $1650^{\circ} \mathrm{C}$ ) en la reacción de formación $\mathrm{Al}_{2} \mathrm{TiO}_{5}$, a partir de la mezcla equimolar de polvos finos (diámetro promedio $<0.5 \mu \mathrm{m}$ ) de alúmina y titania de elevada pureza. Se ha determinado la influencia de las condiciones de tratamiento térmico en el grado de reacción de formación del $\mathrm{Al}_{2} \mathrm{TiO}_{5}$, el tamaño de las celdas de $\mathrm{Al}_{2} \mathrm{TiO}_{5}$ formadas y en la eficiencia de la posterior molienda de los materiales.Los compactos preparados a partir de la mezcla de los polvos, fueron prensados isostaticamente. Parte de los compactos fueron tratados térmicamente utilizando isotermas en el margen de temperaturas de 1350 y $1650^{\circ} \mathrm{C}$. Otros compactos fueron sometidos a un tratamiento térmico en dos etapas, tratados entre 1350 y $1500^{\circ} \mathrm{C}$ y, a continuación se procedió a su molienda, posterior prensado y tratamiento utilizando isotermas a $1600^{\circ} \mathrm{C}$. La evolución de la reacción se estudió mediante las técnicas de difracción de rayos $\mathrm{X}$, microscopía óptica y microscopía electrónica de barrido. El estudio de la eficiencia de molienda se llevo a cabo mediante un analizador de tamaño de partículas láser. Los resultados obtenidos indican la evolución de la reacción desde una matriz no reaccionada en compactos tratados a $1350^{\circ} \mathrm{C}$, hasta la reacción prácticamente completa, salvo la presencia de partículas aisladas de alúmina dentro de la matriz de $\mathrm{Al}_{2} \mathrm{TiO}_{5}$, en compactos tratados a $1650^{\circ} \mathrm{C}$. El tipo de microestructura del $\mathrm{Al}_{2} \mathrm{TiO}_{5}$ formado, determinada por el tratamiento térmico, afecta al posterior proceso de molienda de los materiales de $\mathrm{Al}_{2} \mathrm{TiO}_{5}$.
\end{abstract}

Palabras claves: Titanato de aluminio, reacción en estado sólido, microestructura, molienda

\section{Aluminum titanate formation by sold-state reaction of alumina and titania}

In this work, the formation of $\mathrm{Al}_{2} \mathrm{TiO}_{5}$ from equimolar mixtures of high purity and fine grained $\left(\mathrm{d}_{50}<50 \mu \mathrm{m}\right) \mathrm{Al}_{2} \mathrm{O}_{3}$ and $\mathrm{TiO} \mathrm{O}_{2}$ powders and the influence of the microstructure of the obtained compacts on the milling efficiency have been studied. Different thermal treatments with maximum temperatures between 1350 and $1650^{\circ} \mathrm{C}$ have been considered and their influence in the degree of reaction and the cell size of $\mathrm{Al}_{2} \mathrm{TiO}_{5}$ have been established. Green compacts have been obtained by isostatic pressing. Some compacts have been thermally treated between 1350 and $1650^{\circ} \mathrm{C}$ using a single thermal cycle. The other compacts have been thermally treated in two stages using $1600^{\circ} \mathrm{C}$ as maximum temperature, with an intermediate process of grinding, milling and pressing after the initial low temperature $\left(1350-1500^{\circ} \mathrm{C}\right)$ treatment. The evolution of the reaction has been studied by X-ray diffraction, optical microscopy and scanning electron microscopy. The efficiency of the milling processes has been followed using the evolution of the particle size distribution. The results showed that the degree of reaction changes from practically no reaction at $1350^{\circ} \mathrm{C}$ to the complete reaction at $1650^{\circ} \mathrm{C}$, except for the presence of some isolated alumina particles. The thermal treatment conditions used to obtain the materials determine the obtained microstructures of $\mathrm{Al}_{2} \mathrm{TiO}_{5}$ and hence affect the grinding conditions.

Key word: Aluminum titanate, solid-state reaction, microstructures, grinding.

\section{INTRODUCCIÓN}

La reacción de formación en estado sólido del titanato de aluminio, $\mathrm{Al}_{2} \mathrm{TiO}_{5}$, a partir de la mezcla equimolar de polvos de alúmina y titania ha sido estudiada recientemente por diversos investigadores (1-9). En general, en todos los trabajos se establece que la reacción de formación del $\mathrm{Al}_{2} \mathrm{TiO}_{5}$ envuelve procesos de nucleación y crecimiento que se dan por completo en estado sólido y que el final de la reacción está controlado por la difusión de una o varias especies. La reacción de formación del $\mathrm{Al}_{2} \mathrm{TiO}_{5}$ no sólo implica la energía necesaria para la creación de una nueva superficie, también requiere una energía elástica adicional que supere el constreñimiento elástico que ejerce la matriz no reaccionada de partículas de alúmina y titania sobre los granos de $\mathrm{Al}_{2} \mathrm{TiO}_{5}$ en formación.

La mayoría de los trabajos recientes sobre la reacción de formación del $\mathrm{Al}_{2} \mathrm{TiO}_{5}$ han sido realizados sobre compactos preparados a partir de la mezcla equimolar de polvos de $\mathrm{TiO}_{2}$ de alta pureza obtenidos por vía química y diferentes tipos de polvos de $\mathrm{Al}_{2} \mathrm{O}_{3}$ de distinta naturaleza. En general, se encuentra descrito para este tipo de compactos que la microestructura del $\mathrm{Al}_{2} \mathrm{TiO}_{5}$ que se forma esta caracterizada por una matriz de celdas porosas de $\mathrm{Al}_{2} \mathrm{TiO}_{5}$, separadas por grietas, que contienen en muchos casos inclusiones de $\mathrm{Al}_{2} \mathrm{O}_{3} \mathrm{y} \mathrm{TiO}_{2}$. En compactos tratados a temperaturas superiores a $1500^{\circ} \mathrm{C}$ las celdas presentan menos porosidad y los granos de $\mathrm{Al}_{2} \mathrm{TiO}_{5}$ tienen forma tabular.

En la mayoría de las investigaciones recientes se citan como referencias básicas los trabajos de Freudenberg y Mocellin $(1,2)$. Estos investigadores centran su trabajo en estudios 
sobre compactos preparados a partir de mezclas equimolares de polvos finos de alúmina $\left(\mathrm{d}_{50}<0.4 \mu \mathrm{m}\right)$ y polvos finos $\left(\mathrm{d}_{50}<\right.$ $1.5 \mu \mathrm{m})$ o gruesos $\left(\mathrm{d}_{50}<13 \mu \mathrm{m}\right)$ de titania, sometidos a tratamientos de hasta 100 horas en el margen de temperaturas entre 1297 y $1427^{\circ} \mathrm{C}$. En los trabajos realizados sobre compactos preparados a partir de mezclas equimolares de polvos finos $\left(\mathrm{d}_{50}<0.4 \mu \mathrm{m}\right)$ de alúmina y polvos finos $\left(\mathrm{d}_{50}<1.5 \mu \mathrm{m}\right)$ de titania, tratados en el margen de temperaturas en el cual se inicia la reacción $\left(1297\right.$ a $\left.1327^{\circ} \mathrm{C}\right)$, durante diferentes intervalos de tiempos, se observa el crecimiento aislado de celdas de $\mathrm{Al}_{2} \mathrm{TiO}_{5}$ dentro de una matriz porosa y sin reaccionar de partículas de $\mathrm{Al}_{2} \mathrm{O}_{3}$ y $\mathrm{TiO}_{2}$. Las celdas de $\mathrm{Al}_{2} \mathrm{TiO}_{5}$ son igualmente porosas y en su interior hay inclusiones de $\mathrm{Al}_{2} \mathrm{O}_{3}$ y $\mathrm{TiO}_{2}$. El crecimiento de las celdas de $\mathrm{Al}_{2} \mathrm{TiO}_{5}$ avanza hasta que el volumen de la muestra es completamente ocupado, a partir de este momento la reacción de formación del $\mathrm{Al}_{2} \mathrm{TiO}_{5}$ continúa a velocidades mucho menores (0-10 horas: formación de $5 \%$ peso $\mathrm{Al}_{2} \mathrm{TiO}_{5} /$ hora vs $10-100$ horas: formación de $0.005 \%$ peso $\mathrm{Al}_{2} \mathrm{TiO}_{5} /$ hora). Estos autores observaron que, en los compactos tratados hasta 100 horas a la temperatura máxima, existe aproximadamente el $40 \%$ en peso de los óxidos iniciales sin reaccionar dentro de las celdas de $\mathrm{Al}_{2} \mathrm{TiO}_{5}$.

Freudenberg y Mocellin identificaron dos estados de evolución de la reacción durante estos tratamientos isotérmicos a temperaturas del orden de $1300^{\circ} \mathrm{C}$. La reacción inicial, caracterizada por la nucleación y crecimiento del $\mathrm{Al}_{2} \mathrm{TiO}_{5}$ en puntos aleatorios del compacto, independientemente de la temperatura de tratamiento y, el estado final de la reacción, correspondiente a la eliminación por difusión de las partículas de $\mathrm{Al}_{2} \mathrm{O}_{3}$ y/o $\mathrm{TiO}_{2}$ atrapadas durante el crecimiento inicial de los cristales de $\mathrm{Al}_{2} \mathrm{TiO}_{5}$.

Estos autores propusieron que los puntos aleatorios de nucleación de las celdas de $\mathrm{Al}_{2} \mathrm{TiO}_{5}$ estaban constituidos por impurezas, lo que esta de acuerdo con la teoría clásica de reacción en estado sólido ${ }^{3}$ que establece que, la nucleación de la mayoría de las fases en estado sólido ocurre de forma heterogénea, debido a impurezas, imperfecciones estructurales y/o discontinuidades en los límites de grano, defectos que facilitan la nucleación. La acción de tales sustratos nucleantes es reducir la barrera creada por la energía superficial de la mezcla de óxidos iniciales. Cuando se forma un núcleo sobre un sustrato, aparte de la formación de la interfase "núcleo-matriz", se reemplaza parte de la superficie "matriz-sustrato" de alta energía por una superficie "núcleo-sustrato" de baja energía, resultando, por consiguiente, en una menor contribución a la energía superficial total.

Con el fin de poder estudiar la reacción de formación del $\mathrm{Al}_{2} \mathrm{TiO}_{5}$ a temperaturas mas elevadas, Freudenberg y Mocellin (2) estudiaron compactos preparados a partir de la mezcla equimolar de polvos gruesos $\left(\mathrm{d}_{50}=13 \mu \mathrm{m}\right)$ de titania y polvos finos $\left(\mathrm{d}_{50}<0.4 \mu \mathrm{m}\right)$ de alúmina, de pureza superior a los utilizados en los trabajos anteriores, tratados a temperaturas entre 1297 y $1427^{\circ} \mathrm{C}$. Estos autores determinaron la existencia de dos tipos de nucleación y crecimiento en función del margen de temperatura: a baja temperatura $\left(\mathrm{T}<1327^{\circ} \mathrm{C}\right)$, nucleación y crecimiento caracterizados por un limitado numero de puntos de "nucleación fácil", al igual que ocurría en los compactos preparados a partir de la mezcla de polvos finos y, a alta temperatura $\left(\mathrm{T} \geq 1427^{\circ} \mathrm{C}\right)$, nucleación y crecimiento casi instantáneos a través de todo el compacto. Por lo tanto Freudenberg y Mocellin concluyeron que la nucleación y crecimiento dependen de la temperatura de tratamiento y lo atribuyeron a la barrera de energía elástica que existe para la formación del $\mathrm{Al}_{2} \mathrm{TiO}_{5}$, debido al aumento de volumen que implica la forma- ción de ésta fase. A alta temperatura, la fuerza conductora para la formación del $\mathrm{Al}_{2} \mathrm{TiO}_{5}$ de origen químico es suficiente para sobrepasar esta barrera. Por el contrario, a baja temperatura la barrera de energía elástica solo puede ser sobrepasada en los "puntos de nucleación fácil" que constituyen las discontinuidades del material. Asimismo, observaron que, a baja temperatura, la limitada nucleación permite la existencia de pares de difusión metaestables del tipo $\mathrm{Al}_{2} \mathrm{TiO}_{5} / \mathrm{TiO}_{2} / \mathrm{Al}_{2} \mathrm{O}_{3}$ donde el $\mathrm{TiO}_{2}$ ejerce una doble función como reactivo y camino rápido para la difusión de $\mathrm{Al}$, y a alta temperatura, la rápida nucleación conduce a arreglos del tipo $\mathrm{Al}_{2} \mathrm{O}_{3} / \mathrm{Al}_{2} \mathrm{TiO}_{5} / \mathrm{TiO}_{2}$ que son termodinámicamente estables. Por consiguiente, debido al lento transporte de los de óxidos iniciales a través de las celdas de $\mathrm{Al}_{2} \mathrm{TiO}_{5}$ la velocidad de crecimiento de $\mathrm{Al}_{2} \mathrm{TiO}_{5}$ por difusión es menor a altas temperaturas.

Thomas y Stevens (4) estudiaron la reacción de formación del $\mathrm{Al}_{2} \mathrm{TiO}_{5}$ sobre compactos preparados a partir de mezclas equimolares de polvos del orden de $73 \mathrm{~nm}$ de tamaño, obtenidos por cooprecipitación química de alcóxidos de alúmina y de titania, sometidos a tratamientos térmicos de 2 horas en un margen de temperatura entre 1320 y $1500^{\circ} \mathrm{C}$. Las microestructuras de los compactos preparados por estos autores muestran la formación de celdas porosas de $\mathrm{Al}_{2} \mathrm{TiO}_{5}$ totalmente rodeadas por grietas. Las celdas de $\mathrm{Al}_{2} \mathrm{TiO}_{5}$ poseen una sub-estructura formada por pequeños dominios de cristales de $\mathrm{Al}_{2} \mathrm{TiO}_{5}$ orientados en direcciones preferenciales. De acuerdo con estos autores, el origen de los dominios sería, posiblemente, el arreglo de los aglomerados iniciales dentro de la matriz sin reaccionar y la formación de grietas seria debida a la existencia de zonas de discontinuidad entre los distintos arreglos preferenciales. En estos compactos de $\mathrm{Al}_{2} \mathrm{TiO}_{5}$, preparados a partir de polvos de alta pureza, el tamaño de las celdas de $\mathrm{Al}_{2} \mathrm{TiO}_{5}(\approx$ $100 \mu \mathrm{m})$ es relativamente independiente de la temperatura de tratamiento y, el grado de agrietamiento aumenta con la temperatura.

Buscaglia y col. (5-8) estudiaron el efecto de las características de los polvos de alúmina en la reacción de formación del $\mathrm{Al}_{2} \mathrm{TiO}_{5}$. Los compactos estudiados en estos trabajos fueron preparados a partir de mezclas equimolares de polvos finos $\left(\mathrm{d}_{50}=1 \mu \mathrm{m}\right)$ de titania con polvos de alúmina de distinta granulometria $\left(\mathrm{d}_{50}=0.8,0.5,0.3, \leq 5 \mu \mathrm{m}\right)$, distinta estructura cristalina (fases $\alpha-\gamma$ ), con y sin la adición de $\mathrm{MgO}$, tratados a temperaturas entre 1100 y $1600^{\circ} \mathrm{C}$. En todos los casos, las microestructuras de los compactos preparados por estos autores fueron del mismo tipo que las mostradas en otros trabajos, celdas porosas de $\mathrm{Al}_{2} \mathrm{TiO}_{5}$, separadas o no por grietas y con diferentes contenidos de partículas de $\mathrm{Al}_{2} \mathrm{O}_{3}$ y TiO 2 sin reaccionar, en función de la temperatura de tratamiento. El tamaño alcanzado por las celdas de $\mathrm{Al}_{2} \mathrm{TiO}_{5}$, independiente del tiempo de permanencia a la temperatura máxima de tratamiento, corrobora la hipótesis de Freudenberg y Mocellin referente a que, una vez que el volumen de las muestras es completamente ocupado por celdas de $\mathrm{Al}_{2} \mathrm{TiO}_{5}$, el crecimiento de las celdas es bastante lento debido a la existencia de pares de nucleación del tipo $\mathrm{Al}_{2} \mathrm{O}_{3} / \mathrm{Al}_{2} \mathrm{TiO}_{5} / \mathrm{TiO}_{2}$.

Buscaglia y col. (5-8) concluyen que la naturaleza de los polvos de alúmina utilizados en la reacción, determina la evolución microestructural de las celdas de $\mathrm{Al}_{2} \mathrm{TiO}_{5}$ formadas. Por una parte, al disminuir el diámetro de las partículas de alúmina aumenta el tamaño de las celdas de $\mathrm{Al}_{2} \mathrm{TiO}_{5}\left(0.8 \mu \mathrm{m} \mathrm{Al}_{2} \mathrm{O}_{3}\right.$ $100 \mu \mathrm{m} \mathrm{Al} \mathrm{TiO}_{5}$ vs $0,3 \mu \mathrm{m} \mathrm{Al}_{2} \mathrm{O}_{3}-400 \mu \mathrm{m} \mathrm{Al}_{2} \mathrm{TiO}_{5}$ ) y, por otra, la presencia de $\mathrm{MgO}$ en la mezcla de óxidos iniciales hace que disminuya el tamaño de las celdas de $\mathrm{Al}_{2} \mathrm{TiO}_{5}(\approx 200 \mu \mathrm{m}$ sin $\mathrm{MgO}$ vs $\approx 20 \mu \mathrm{m}$ con $\mathrm{MgO}$ ). 
Las observaciones de Wohlfromm (9) sobre compactos preparados a partir de mezclas equimolares de polvos finos $\left(\mathrm{d}_{50}=\right.$ $0.45 \mu \mathrm{m})$ de alúmina y polvos finos $\left(\mathrm{d}_{50}=0.45 \mu \mathrm{m}\right)$ de titania, tratados a temperaturas $\leq 1550^{\circ} \mathrm{C}$, corroboran los trabajos de Freudenberg $(1,2)$ y Buscaglia (5-8) en lo referente a la reacción de formación del $\mathrm{Al}_{2} \mathrm{TiO}_{5}$ a baja temperatura. Este autor observó que, en materiales tratados a temperaturas entre 1350 y $1450^{\circ} \mathrm{C}$, están presentes cantidades notables de óxidos sin reaccionar, en forma de finas partículas intraganulares atrapadas en las celdas porosas de $\mathrm{Al}_{2} \mathrm{TiO}_{5}$. La eliminación de estas partículas a la temperatura de $1550^{\circ} \mathrm{C}$ requiere tiempos de tratamiento muy largos. En materiales tratados a $1550^{\circ} \mathrm{C}$ Wohlfromm observó, al igual que Stevens (3) la formación de celdas porosas de $\mathrm{Al}_{2} \mathrm{TiO}_{5}$ rodeadas de grietas, de tamaños aproximados de $100 \mu \mathrm{m}$. Este autor estudió la influencia de la velocidad de tratamiento térmico en el tamaño de las celdas de $\mathrm{Al}_{2} \mathrm{TiO}_{5}$ formadas, observando que, si bien el tamaño de las celdas de $\mathrm{Al}_{2} \mathrm{TiO}_{5}$ puede ser reducido utilizando velocidades de calentamiento rápidas, este tipo de tratamientos va en detrimento del grado de reacción.

El propósito del presente trabajo ha sido la obtención de polvos finos de titanato de aluminio, $\mathrm{Al}_{2} \mathrm{TiO}_{5}$, de elevada pure$\mathrm{za}$ a fin de introducirlos como fase secundaria en matrices de $\mathrm{Al}_{2} \mathrm{O}_{3}-\mathrm{Al}_{2} \mathrm{TiO}_{5}$, en las cuales no se produzca agrietamiento debido a la presencia del $\mathrm{Al}_{2} \mathrm{TiO}_{5}$. Para ello, es preciso optimizar tanto el grado de reacción de los óxidos iniciales como la eficiencia de la molienda posterior, para obtener polvos de $\mathrm{Al}_{2} \mathrm{TiO}_{5}$ de granulometria fina y homogénea. En principio, la eficiencia de la molienda podría verse incrementada por un tamaño pequeño de las celdas de $\mathrm{Al}_{2} \mathrm{TiO}_{5}$, una alta porosidad o un alto grado de agrietamiento. A partir de los resultados discutidos anteriormente, el único de estos tres factores que puede modificarce, utilizando tratamientos con una sola isoterma, sin perder un alto grado de reacción, es el grado de agrietamiento incrementando la temperatura máxima de tratamiento.

En este trabajo se ha estudiado el efecto que tiene un tratamiento térmico en dos etapas, con molienda intermedia y prensado, tanto en el grado de reacción, como en la microestructura de los materiales obtenidos. En paralelo, se han estudiado materiales obtenidos vía tratamientos térmicos en una sola etapa y se ha determinado el efecto del tipo de tratamiento en el grado de reacción y la eficiencia de la molienda de los materiales obtenidos.

\section{PROCEDIMIENTO EXPERIMENTAL}

Las materias primas utilizadas en este estudio son polvos comerciales de alúmina ${ }^{\circ}\left(\alpha-\mathrm{Al}_{2} \mathrm{O}_{3}\right)$ y titania ${ }^{*}\left(\mathrm{TiO}_{2}\right)$ de alta pureza. De acuerdo con las especificaciones de los fabricantes (\% en peso) la alúmina tiene una pureza superior al $99.9 \%$ y presenta como impurezas principales el $\mathrm{SiO}_{2}, \mathrm{Fe}_{2} \mathrm{O}_{3}$ y $\mathrm{Na}_{2} \mathrm{O}$ en proporciones del orden de $0.005 \%$ y $\mathrm{MgO}, \mathrm{CaO}$ en proporciones inferiores al $0.001 \%$, la titania tiene una pureza superior al $99.4 \%$ y presenta como impurezas principales sales ionicas $(\approx 0.005 \%)$ y contenidos de $\mathrm{Fe}_{2} \mathrm{O}_{3}$ menores del $0.014 \%$. La superficie especifica de los polvos fue determinada por el método BET de adsorción de $\mathrm{N}_{2}$ (Monosorb, Quantachrome Corporation, EE UU). Para determinar la distribución del tamaño de partícula se utilizó un analizador de tamaño de partículas láser (Mastersizer-S, Malvern Instruments, Reino Unido).

${ }^{\circ}$ Condea HPA-0.5, Tucson, Arizona, EE UU.

* Merck 808, Darmstadt, Alemania.
Se realizó una mezcla equimolar de los polvos de $\mathrm{Al}_{2} \mathrm{O}_{3}$ y $\mathrm{TiO}_{2}$ en un molino de bolas durante 4 horas utilizando como medio alcohol isopropílico. La mezcla fue posteriormente secada durante 12 horas a $60^{\circ} \mathrm{C}$ y tamizada $(63 \mu \mathrm{m})$. La compactación del polvo se lleva a cabo por prensado isostático a 200 MPa obteniéndose barras cilíndricas de $5 \mathrm{~mm}$ de diámetro, a estos compactos en verde se les denominará a partir de ahora AT-V.

Los compactos AT-V fueron sometidos a tratamientos térmicos en aire en un horno eléctrico con resistencias de disiliciuro de molibdeno (Superkanthal, $1700^{\circ} \mathrm{C}$, Suiza), en el margen de temperaturas entre 1350 y $1650^{\circ} \mathrm{C}$, utilizando siempre velocidades de calentamiento y enfriamiento de $5^{\circ} \mathrm{C} / \mathrm{min}$ y 3 horas de permanencia a la temperatura máxima. A los compactos AT-V sometidos a un tratamiento térmico simple se les denominará AT-temperatura donde: temperatura $=1350,1400$, $1500,1600,1650^{\circ} \mathrm{C}$ y a los compactos AT-V sometidos a un tratamiento térmico en dos etapas, con un proceso intermedio de molienda y prensado isostático a $200 \mathrm{MPa}$, se les denominará AT-temperatura ${ }_{1}$-temperatura 2 donde: temperatura ${ }_{1}$ temperatura ${ }_{2}=1350-1600,1400-1600,1500-1600^{\circ} \mathrm{C}$. Las densidades de los compactos en verde y tratados térmicamente, se determinaron por el método de Arquímedes utilizando siempre como fluido mercurio.

Para la determinación de las fases cristalinas presentes se utilizó la difracción de rayos X sobre polvos (D5000, Siemens AG, Alemania) empleando la radiación $\mathrm{K}_{\alpha}$ del cobre $=1,54051 \AA$ ) y los difractogramas obtenidos se procesaron utilizando las fichas ASTM: 26-0040 $\left(\mathrm{Al}_{2} \mathrm{TiO}_{5}\right), 42-1468\left(\mathrm{Al}_{2} \mathrm{O}_{3}\right)$, 21-1276 $\left(\mathrm{TiO}_{2}\right)$. Los espectros se registraron para ángulos de difracción, ${ }^{\circ} 2 \theta$, entre 25 y $28^{\circ} 2 \theta$, ya que en esa zona se encuentran los picos no coincidentes y de mayores intensidades del $\mathrm{Al}_{2} \mathrm{TiO}_{5}$ (022), $\mathrm{Al}_{2} \mathrm{O}_{3}(012), \mathrm{TiO}_{2}$ (110). Las muestras objeto de estudio fueron siempre molidas en un mortero de wolframio y tamizadas hasta un tamaño de partícula inferior de $37 \mu \mathrm{m}$.

Para la caracterización microestructural, se utilizó microscopía óptica de luz reflejada (D-7082, Zeiss, Alemania) y microscopía electrónica de barrido (DSM-950, Zeiss, Alemania) equipada con una unidad de microanálisis por dispersión de energías de rayos X (EDX). Las muestras a objeto de estudio fueron pulidas con polvo de diamante hasta $1 \mu \mathrm{m}$.

Los compactos seleccionados por su mayor grado de reacción de formación de $\mathrm{Al}_{2} \mathrm{TiO}_{5}$ y tipo de microestructura de $\mathrm{Al}_{2} \mathrm{TiO}_{5}$, fueron sometidos a un proceso de trituración, tamizado $(100 \mu \mathrm{m})$ y de molienda en atrición, utilizando como medio alcohol isopropílico, durante diferentes periodos de tiempo y, se tomaron muestras directamente del proceso de molienda para determinar la distribución de tamaños de partículas, con el analizador de tamaños de partículas láser.

\section{RESULTADOS}

La caracterización general de los polvos de partida se encuentra en la tabla I. Se puede observar que las materias primas utilizadas poseen características físicas similares en cuanto a la superficie específica y el tamaño de partícula, siendo ambos parámetros ligeramente inferiores en el caso de la titania.

Los valores de la densidad de los compactos en verde y tratados térmicamente se recogen en la tabla II. Se observa que todos los compactos tratados entre 1350 y $1500^{\circ} \mathrm{C}$ presentan valores coincidentes de densidad. Asimismo, los compactos 
TABLA I: CARACTERÍSTICAS GENERALES DE LOS POLVOS INICIALES.

\begin{tabular}{|c|c|c|}
\hline $\begin{array}{l}\text { CARACTERISTICAS } \\
\text { Polvos iniciales }\end{array}$ & $\begin{array}{c}\mathrm{AL}_{2} \mathrm{O}_{3} \\
\text { Condea HPA-0.5 }\end{array}$ & $\begin{array}{c}\mathrm{TiO}_{2} \\
\text { Merck 808 }\end{array}$ \\
\hline Constitución mineralógica & Corindón & Anatasa \\
\hline Superficie específica $\left(\mathrm{m}^{2} / \mathrm{g}\right)$ : & $10 \pm 0.5$ & $9 \pm 0.5$ \\
\hline Tamaño de partícula $(\mu \mathrm{m}): \quad d_{90}$ & 0.76 & 0.61 \\
\hline $\mathrm{d}_{50}$ & 0.40 & 0.30 \\
\hline $\mathrm{d}_{10}$ & 0.27 & 0.19 \\
\hline
\end{tabular}

TABLA II: DENSIDADES OBTENIDAS EN LOS COMPACTOS.

\begin{tabular}{c|c}
\hline Compactos & Densidad $\pm \mathbf{0 . 0 2}\left(\mathrm{g} / \mathbf{c m}^{3}\right)$ \\
\hline AT- V & 2.32 \\
\hline AT-1350 a AT-1500 & 2.75 \\
\hline AT-1600 a AT-1650 & 2.84 \\
\hline AT-1350-1600 a AT-1500-1600 & 2.98 \\
\hline
\end{tabular}

tratados entre 1600 y $1650^{\circ} \mathrm{C}$ presentan valores coincidentes de densidad, mayores que en el caso anterior. Los valores de la densidad de todos los compactos tratados térmicamente en dos etapas son iguales y superiores al resto.

La figura 1 muestra la evolución mineralógica de todos los materiales en función de la temperatura de tratamiento. En los difractogramas correspondientes a los compactos AT-1350 se observa el pico de $\mathrm{Al}_{2} \mathrm{TiO}_{5}$ y los picos característicos de $\mathrm{Al}_{2} \mathrm{O}_{3}$ y $\mathrm{TiO}_{2}$. Cuando se aumenta la temperatura de tratamiento en los compactos AT-1400 y AT-1500 se observa el pico de $\mathrm{Al}_{2} \mathrm{TiO}_{5}$ y los picos de $\mathrm{Al}_{2} \mathrm{O}_{3}$ y $\mathrm{TiO}_{2}$ de menor intensidad que en los compactos tratados a $1350^{\circ} \mathrm{C}$. En los compactos AT-1600 y AT-1650 se observa únicamente el pico correspondiente al $\mathrm{Al}_{2} \mathrm{TiO}_{5}$. En la figura 2 se recogen las curvas de difracción de rayos $\mathrm{X}$ de los compactos AT-temperatura ${ }_{1}$-temperatura ${ }_{2}$, en las cuales, sólo se observa el pico característico del $\mathrm{Al}_{2} \mathrm{TiO}_{5}$.

En la figura 3 a se muestran las coloraciones observadas en microscopía electrónica de barrido (MEB), utilizando la señal proporcionada por los electrones retrodispersados,

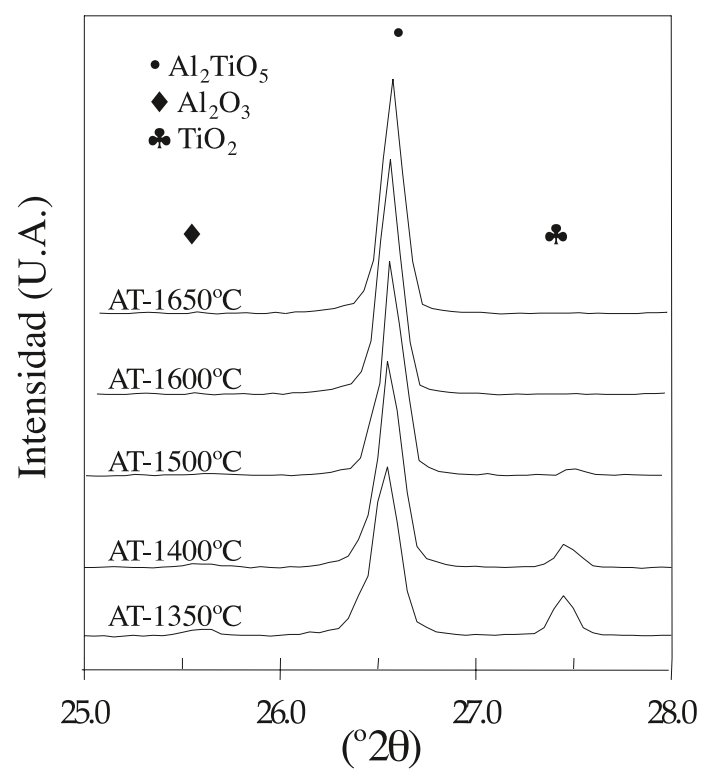

Figura 1. Difractogramas de materiales de $\mathrm{Al}_{2} \mathrm{TiO}_{5}$ obtenidos a partir de un ciclo térmico simple, en aire, a diferentes temperaturas, durante 3 horas.

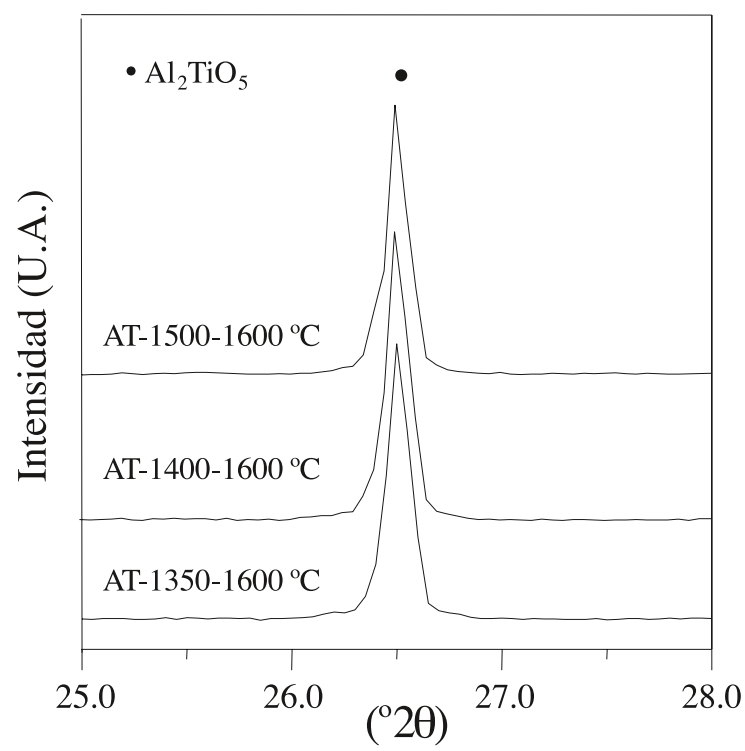

Figura 2. Difractogramas de materiales de $\mathrm{Al}_{2} \mathrm{TiO}_{5}$ obtenidos a partir de un ciclo térmico en dos etapas, con un proceso intermedio de molienda y prensado isostático, tratados en aire, a diferentes temperaturas, durante 3 horas.

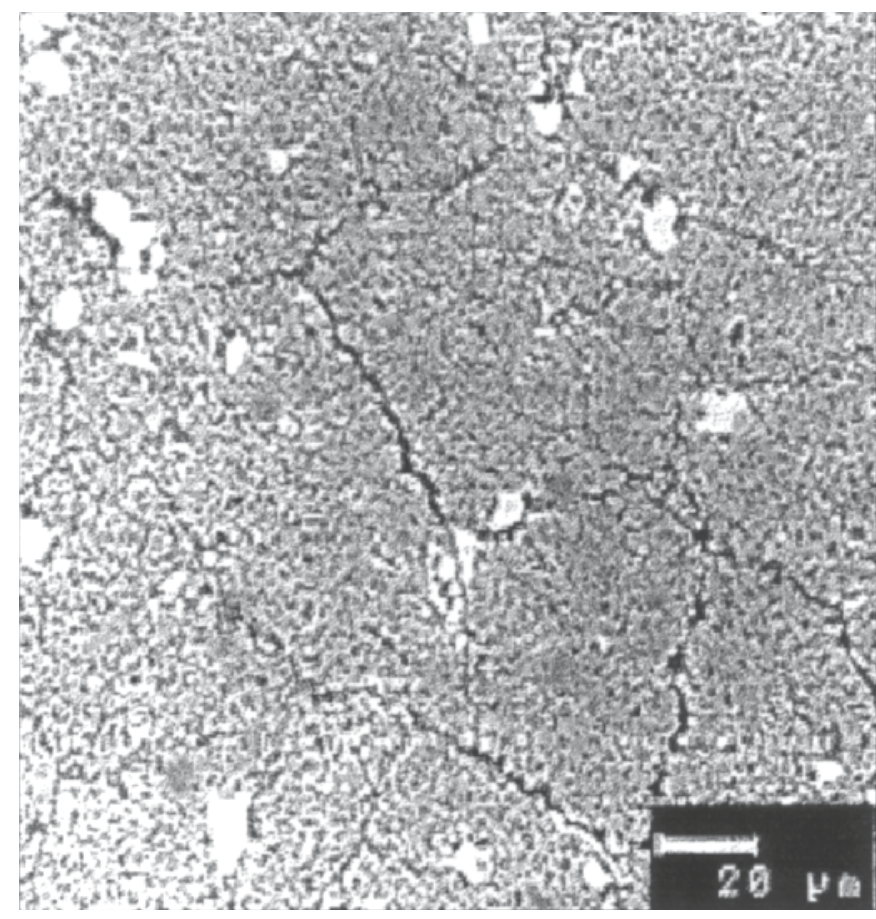

Figura 3a. Micrografía de MEB de materiales de $\mathrm{Al}_{2} \mathrm{TiO}_{5}$, obtenidos a partir de un ciclo térmico simple a $1350^{\circ} \mathrm{C}$, en aire, durante 3 horas.

para las fases presentes en los compactos tratados térmicamente $y$, en la figura $3 \mathrm{~b}$ se recogen las curvas correspondientes obtenidas por microanálisis EDX. Las zonas de color gris que dominan toda la micrografia de la figura 3a corresponden a la fase de $\mathrm{Al}_{2} \mathrm{TiO}_{5}$, las zonas puntuales de color blanco corresponden a la fase de $\mathrm{TiO}_{2}$ y las zonas puntuales de color gris oscuro corresponden a la $\mathrm{Al}_{2} \mathrm{O}_{3}$. Estas mismas fases se observaron, en mayor o menor proporción, en todos los compactos estudiados. 


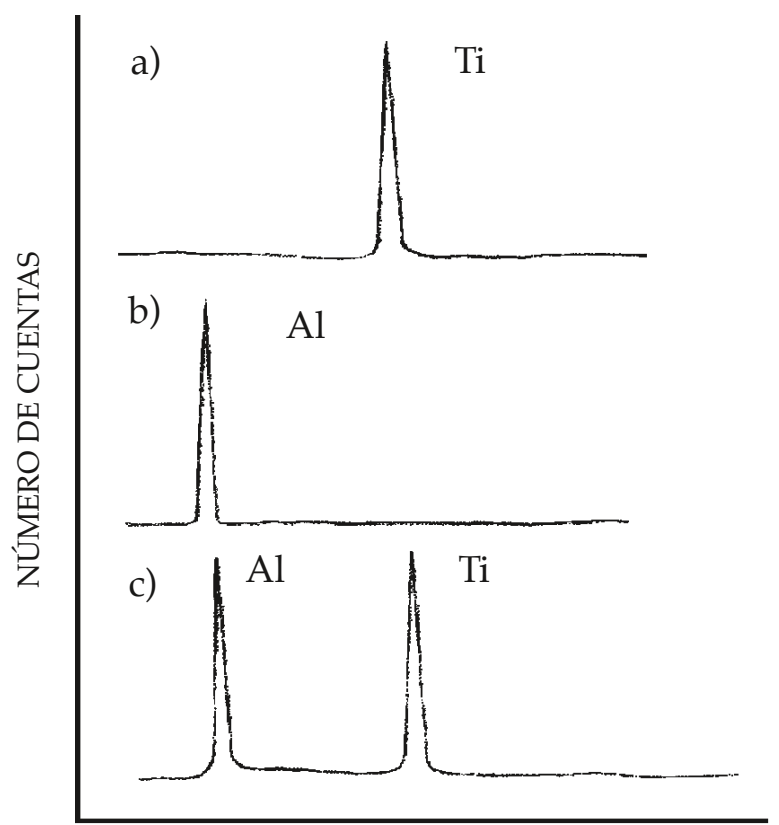

ENERGIA / Kev

Figura 3b. Curvas de microanálisis puntual EDX en materiales $\mathrm{Al}_{2} \mathrm{TiO}_{5}$, figura3a: a)zonas puntuales de color blanco, b) zonas puntuales de color gris oscuro, c) zona de color gris, domina toda la micrografía.
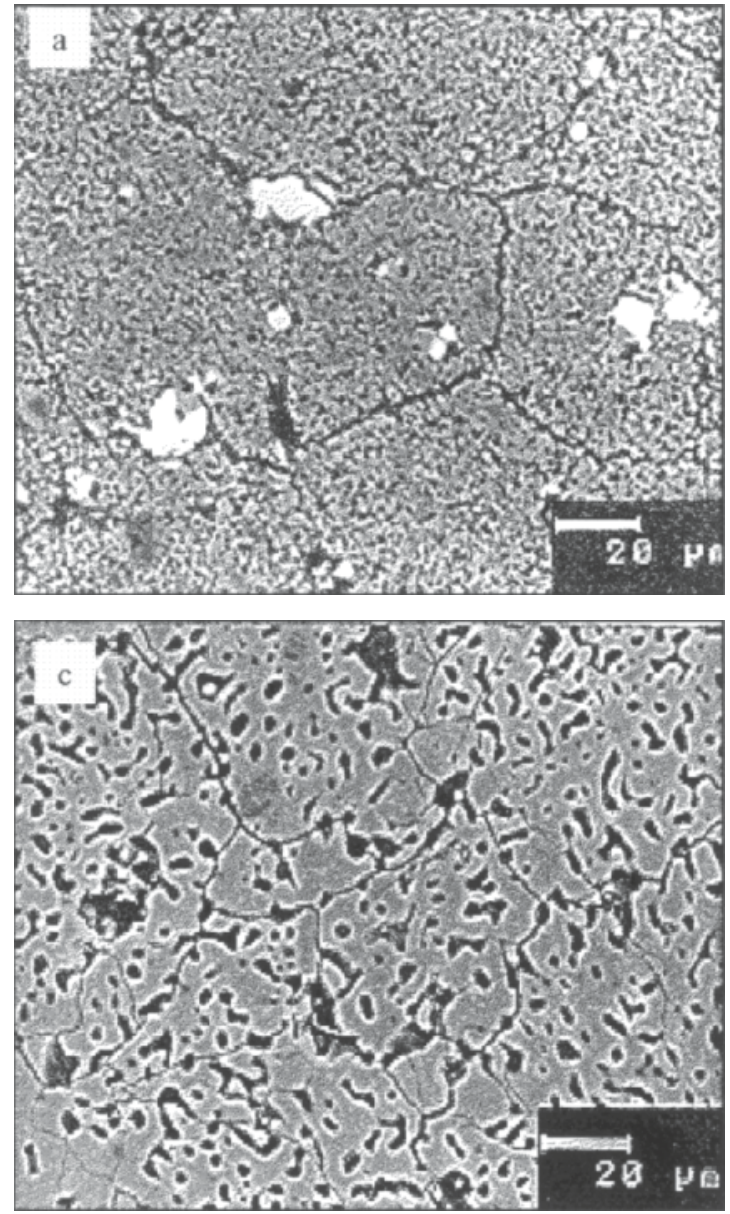

En la figura 4 se muestran las micrografias de MEB obtenidas mediante electrones retrodispersados, donde se observa el efecto de un ciclo térmico simple en la microestructura de materiales de $\mathrm{Al}_{2} \mathrm{TiO}_{5}$ obtenidos.

En los compactos AT-1350 y AT-1400 (figuras 3a-4a) se observa una matriz de celdas porosas de $\mathrm{Al}_{2} \mathrm{TiO}_{5}$ del orden de $60 \mathrm{a}$ $80 \mu \mathrm{m}$ separadas por grietas. Las celdas de $\mathrm{Al}_{2} \mathrm{TiO}_{5}$ contienen en su interior partículas de $\mathrm{Al}_{2} \mathrm{O}_{3}$ de diámetros del orden de 2 $\mu \mathrm{m}$ y partículas de $\mathrm{TiO}_{2}$ de diámetros del orden de 5 a $8 \mu \mathrm{m}$, localizadas en el interior y en los bordes de las celdas.

En los compactos AT-1500 (figura 4b) se observa el mismo tipo de microestructura de celdas porosas de $\mathrm{Al}_{2} \mathrm{TiO}_{5}$, en este caso de tamaño superior $(80-100 \mu \mathrm{m})$ separadas por grietas. Las celdas de $\mathrm{Al}_{2} \mathrm{TiO}_{5}$ contienen menor cantidad de inclusiones de $\mathrm{Al}_{2} \mathrm{O}_{3}$ y TiO 2 que en los compactos tratados a temperaturas inferiores.

En los compactos AT-1600 (figura 4c) no se diferencian las celdas de $\mathrm{Al}_{2} \mathrm{TiO}_{5}$, se observa una matriz de $\mathrm{Al}_{2} \mathrm{TiO}_{5}$, menos porosa que las celdas presentes en los compactos tratados a temperaturas inferiores, con grandes grietas alargadas. Dentro de la matriz de $\mathrm{Al}_{2} \mathrm{TiO}_{5}$ no se observa partículas de $\mathrm{TiO}_{2}$, hay granos de $\mathrm{Al}_{2} \mathrm{O}_{3}$ del orden de 1 a $2 \mu \mathrm{m}$, con bordes poco definidos y algunas partículas de mayor tamaño $(\approx 5-8 \mu \mathrm{m})$.

Los compactos AT-1650 (figura 4d) presentan el mismo tipo de microestructura que los compactos AT-1600. Dentro de la matriz de $\mathrm{Al}_{2} \mathrm{TiO}_{5}$ no se detectan partículas de $\mathrm{TiO}_{2}$, hay granos de $\mathrm{Al}_{2} \mathrm{O}_{3}$ del orden de 1 a $2 \mu \mathrm{m}$, con bordes poco definidos y algunas partículas grandes $(\approx 5-8 \mu \mathrm{m})$ localizadas en las grietas.
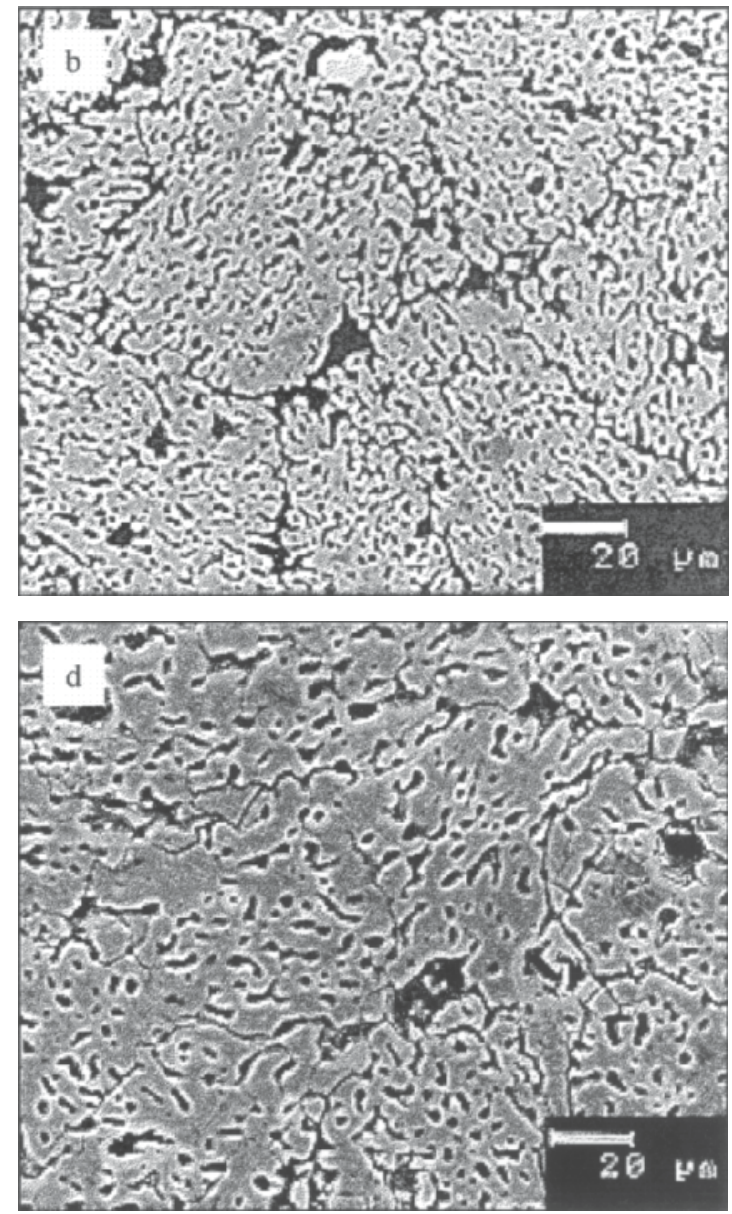

Figura 4. Micrografía de MEB de materiales de $\mathrm{Al}_{2} \mathrm{TiO}_{5}$ obtenidos a partir de un ciclo térmico simple, en aire, durante 3 horas. Se muestra la influencia de un tratamiento intermedio de molienda y prensado en la reacción de formación de $\mathrm{Al}_{2} \mathrm{TiO}_{5}:$ a) AT- $1400^{\circ} \mathrm{C}$, b) $\left.\left.\mathrm{AT}-1500^{\circ} \mathrm{C}, \mathrm{c}\right) \mathrm{AT}-1600^{\circ} \mathrm{C}, \mathrm{d}\right) \mathrm{AT}-1650^{\circ} \mathrm{C}$. 

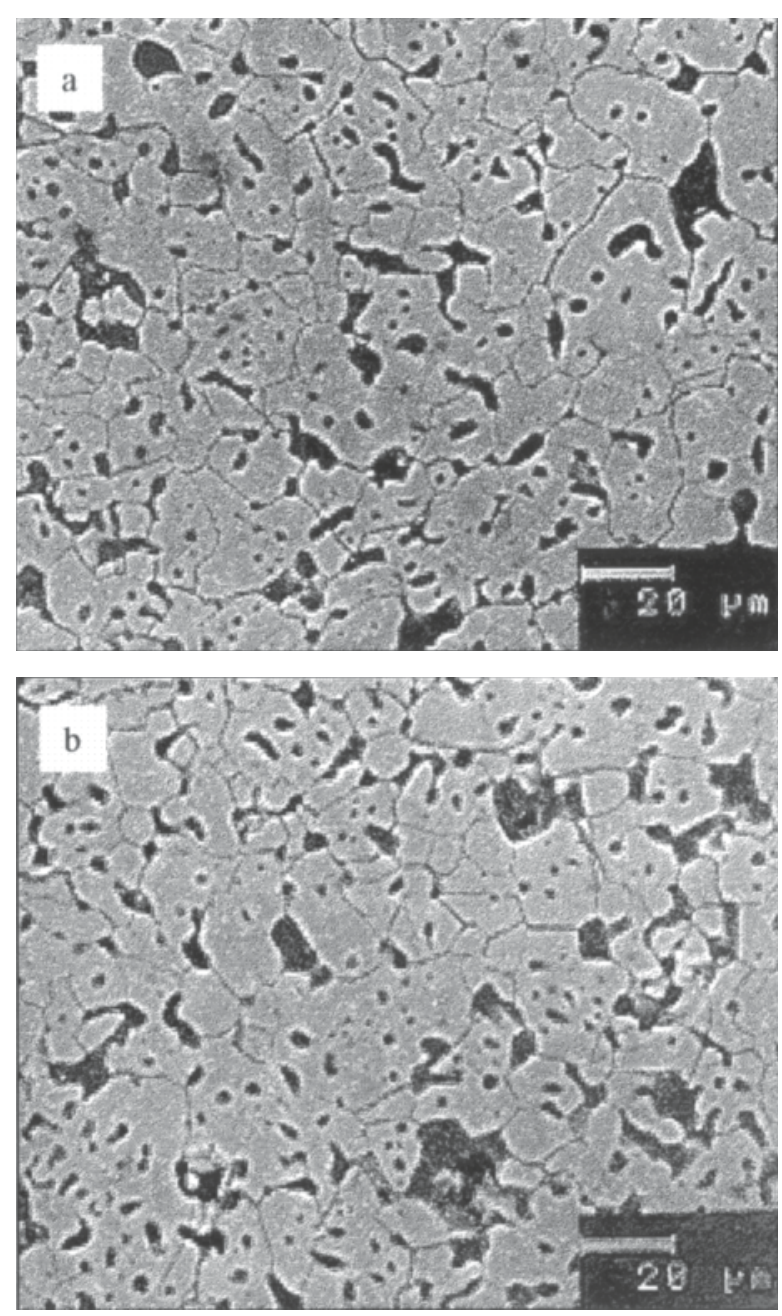

Figura 5. Micrografía de MEB de materiales de $\mathrm{Al}_{2} \mathrm{TiO}_{5}$ obtenidos a partir de un ciclo térmico en dos etapas, en aire, durante tres horas. Se muestra la influencia de un tratamiento intermedio de molienda y prensado en la reacción de formación de $\mathrm{Al}_{2} \mathrm{TiO}_{5}$ : a) AT-1400 $1600^{\circ} \mathrm{C}$, b) AT- $1500-1600^{\circ} \mathrm{C}$.

En las micrografias de MEB de la figura 5 se muestra el efecto de un ciclo térmico, en dos etapas, en la microestructura de los compactos. En todas las micrografias se observa una matriz de granos porosos de $\mathrm{Al}_{2} \mathrm{TiO}_{5}$ del orden de $20 \mu \mathrm{m}$, separados por grietas y algunas partículas de $\mathrm{Al}_{2} \mathrm{O}_{3}$ del orden de 1 a $2 \mu \mathrm{m}$ dispersas dentro de la matriz.

En las curvas de las figuras 6 se recoge la evolución granulométrica en función del tiempo de molienda de atrición, de los compactos tratados térmicamente. Cuando los polvos de $\mathrm{Al}_{2} \mathrm{TiO}_{5}$ son sometidos durante varias horas al proceso de molienda en atrición, las curvas de distribuciones de tamaños de partículas de los polvos AT-1650 presentan valores de distribución inferiores a la de los polvos AT-1400-1600.

En la figura 6a se observa que después un proceso de trituración y tamizado (malla $100 \mu \mathrm{m}$ ) de los compactos AT-1650 y AT-1400-1600: los polvos de $\mathrm{Al}_{2} \mathrm{TiO}_{5}$ obtenidos a partir de los compactos AT-1650 presentan una distribución normal de tamaño de partículas y un $\mathrm{d}_{50} \approx 54 \mu \mathrm{m}$ y, los polvos obtenidos a partir de los compactos AT-1400-1600 presentan una distribución normal de tamaños de partículas ligeramente inferior y un $\mathrm{d}_{50} \approx 38 \mu \mathrm{m}$.

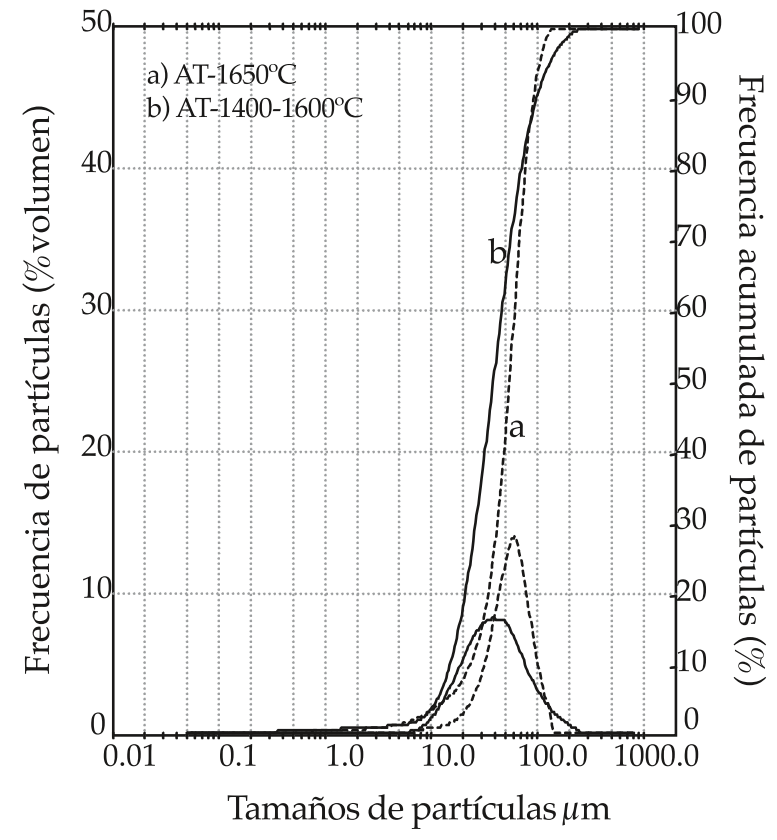

Figura 6a. Distribución granulométrica de compactos de $\mathrm{Al}_{2} \mathrm{TiO}_{2}$, triturados y tamizados (malla $100 \mu \mathrm{m}$ ): a) compactos tratados con un ciclo térmico simple; b) compactos tratados con un ciclo térmico en dos etapas.

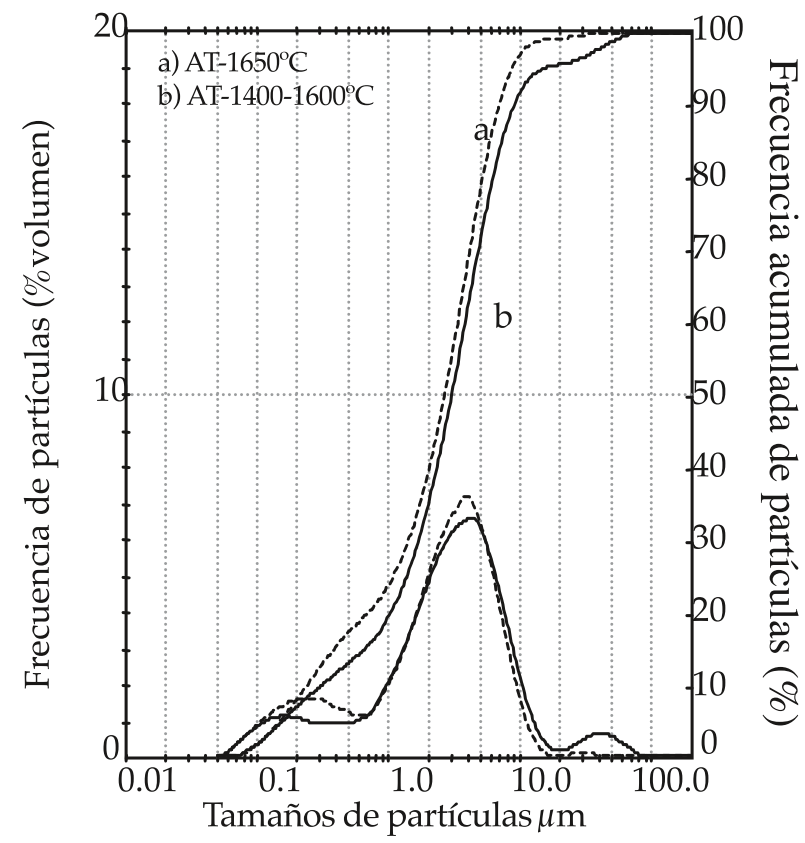

Figura 6b. Distribución granulométrica de polvos de $\mathrm{Al}_{2} \mathrm{TiO}_{5}$, con 1 hora de molienda atrición: a)compactos tratados con un ciclo térmico simple; b) compactos tratados con un ciclo térmico en dos etapas.

En la figura $6 \mathrm{~b}$ se observa que después de una hora de molienda de atrición: los polvos de AT-1650 presentan una distribución bimodal de tamaño de partículas y un $\mathrm{d}_{50} \approx 3 \mu \mathrm{m}$ y, los polvos de AT-1400-1600 presentan una distribución normal de tamaños de partículas, con un $\mathrm{d}_{50} \approx 6 \mu \mathrm{m}$.

En la figura 6c se observa que a partir de dos horas de molienda de atrición: los polvos AT-1650 presentan una distribución bimodal de tamaños de partículas, desplazada hacia tamaños máximos inferiores a $65 \mu \mathrm{m}$, con un $\mathrm{d}_{50} \approx 2.6 \mu \mathrm{m}$ y, los polvos AT-1400-1600 presentan una distribución trimodal de 


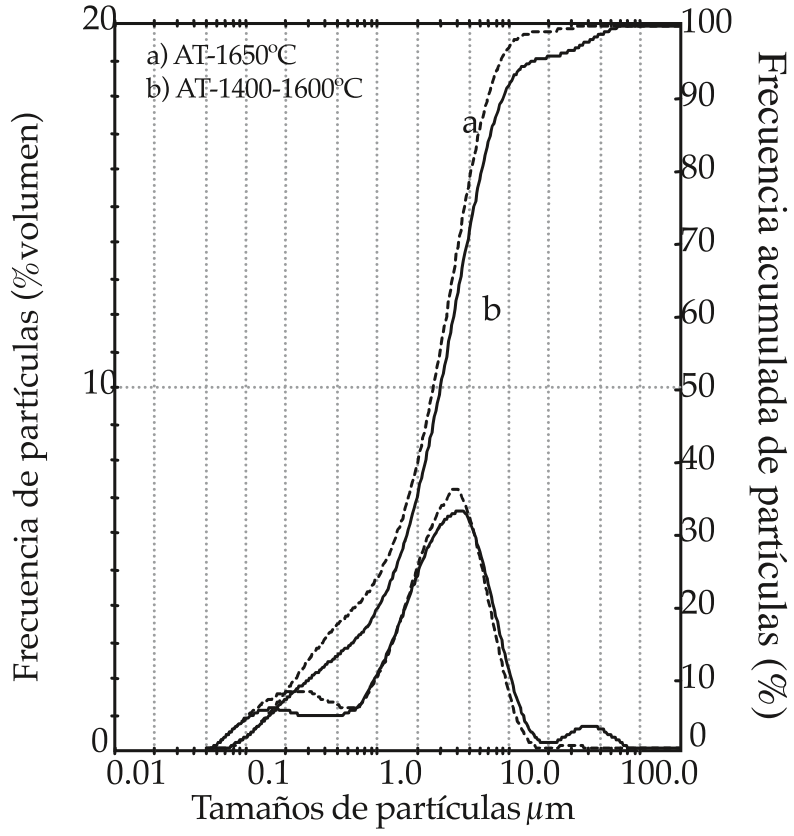

Figura 6c. Distribución granulométrica de polvos de $\mathrm{Al}_{2} \mathrm{TiO}_{5}$, con 2 horas de molienda atrición: a) compactos tratados con un ciclo térmico simple; b) compactos tratados con un ciclo térmico en dos etapas.

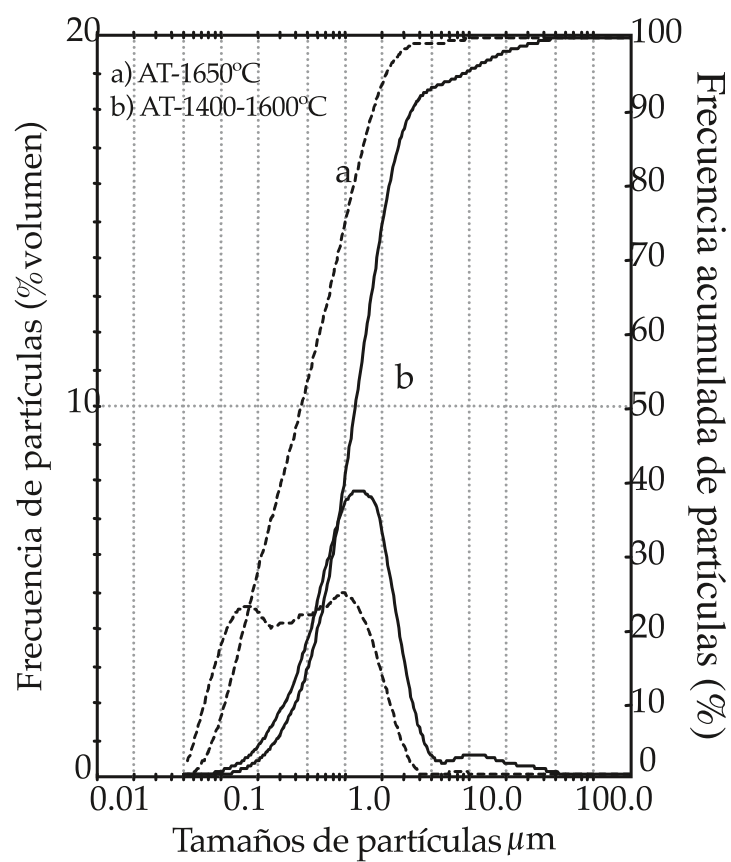

Figura 6d. Distribución granulométrica de polvos de $\mathrm{Al}_{2} \mathrm{TiO}_{5}$, con 8 horas de molienda atrición: a) compactos tratados con un ciclo térmico simple; b) compactos tratados con un ciclo térmico en dos etapas.

tamaños de partículas, desplazada hacia tamaños máximos inferiores a $110 \mu \mathrm{m}$, con un $\mathrm{d}_{50} \approx 3 \mu \mathrm{m}$.

En la figura $6 \mathrm{~d}$ se observa que después de 8 horas de molienda de atrición: los polvos de AT-1650 presentan una distribución bimodal de tamaños de partículas, desplazada hacia tamaños máximos inferiores a $23 \mu \mathrm{m}$, con un $\mathrm{d}_{50} \approx 0.5 \mu \mathrm{m}$ y, los polvos AT-1400-1600 presentan una distribución trimodal de tamaños de partículas, desplazada hacia tamaños máximos inferiores a $80 \mu \mathrm{m}$, con un $\mathrm{d}_{50} \approx 1.2 \mu \mathrm{m}$.

\section{DISCUSION}

Las materias primas utilizadas en este estudio tienen características físicas y químicas (tabla I) similares a las de las utilizadas por Freudenberg (1-2), Buscaglia (5-8) y Wohlfromm (9) en sus investigaciones, ya que se pueden incluir en el grupo de polvos finos $\left(\mathrm{d}_{50} \leq 1 \mu \mathrm{m}\right)$ y de alta pureza. ( $>99.5 \%$ en peso).

El proceso total de reacción formación del $\mathrm{Al}_{2} \mathrm{TiO}_{5}$ consta de tres etapas: la sinterización de la mezcla de óxidos iniciales, que produce el primer aumento de la densidad del material, la formación de las celdas $\mathrm{Al}_{2} \mathrm{TiO}_{5}$, acompañada de una expansión del $11 \%$ en volumen, y la sinterización del $\mathrm{Al}_{2} \mathrm{TiO}_{5}$, que da lugar a la densificación final del material (6).

La densidad en verde alcanzada por los compactos preparados en este trabajo (tabla II) es del orden del $60 \%$ de la densidad teórica, para la mezcla de alúmina y titania, superior a las correspondientes a trabajos anteriores (1-9) (49 a $54 \%$ de la densidad teórica). En principio esta alta densidad en verde podría, favorecer la reacción de formación del $\mathrm{Al}_{2} \mathrm{TiO}_{5}$ debido a una mayor área de contacto sólido/sólido.

A pesar de las diferencias en los valores de densidad en verde, las densidades alcanzadas por los compactos tratados a baja temperatura (AT-1350 a AT-1500, tabla II), son del mismo orden que las de los materiales preparados por Buscaglia (5-8) $\left(2.71-2.76 \mathrm{~g} / \mathrm{cm}^{3}\right)$. En este margen de temperatura la reacción no es completa, como muestran los espectros de difracción de rayos $\mathrm{X}$ (figura 1), en los que se aprecian los picos de los reactivos, y las microestructuras de los materiales obtenidos (figuras $3 a, 4 a, 4 b)$, en las que se observan las partículas de alúmina y titania. Un mayor grado de reacción implica una mayor perdida de densidad en el material, debido a la variación del volumen que conlleva $(\approx 11 \%)$, lo que da lugar a la coincidencia entre los valores de la densidad de los compactos aquí estudiados y los mostrados(5-8).

El que tanto las microestructuras (figuras 3a,4a), como los valores de densidad alcanzados por los compactos AT-1350 y AT-1400, para los cuales el grado de reacción es similar (figura 1), sean coincidentes corrobora la hipótesis de Freudenberg y Mocellin (2), la cual establece que, a temperaturas superiores a la temperatura de formación del $\mathrm{Al}_{2} \mathrm{TiO}_{5}$, las celdas de $\mathrm{Al}_{2} \mathrm{TiO}_{5}$ se forman casi instantáneamente, alcanzándose los máximos de reacción cuando el volumen de la muestra es completamente ocupado.

En el material tratado a $1500^{\circ} \mathrm{C}$ (AT-1500), las celdas de $\mathrm{Al}_{2} \mathrm{TiO}_{5}$ son más densas que en los tratados a temperaturas inferiores (figura 4b), pero el mayor grado de reacción (figura 1) hace que la densidad final alcanzada sea inferior (tabla II). En los compactos tratados a temperaturas superiores (AT-1600 y AT-1650), o con un tratamiento térmico en dos etapas (AT-temperatura -tempertura $_{2}$ ), la reacción es prácticamente completa (figuras 1 y 2), dentro del margen de error experimental asociado con la técnica de difracción de rayos X $(\approx 2-5 \%)$. De hecho, en las microestructuras de estos materiales solo se observan algunos granos aislados de alúmina (figuras 4c,4d y 5). Estos resultados no se han podido contrastar con las investigaciones anteriores (1-9), ya que en ellas no se muestran micrografias de materiales tratados a temperaturas superiores a $1550^{\circ} \mathrm{C}$ y no se estudian los procesos que se suceden a temperaturas superiores a $1600^{\circ} \mathrm{C}$.

En este estudio se pudo observar que cuando los compactos AT-temperatura -temperatura $_{2}$, son sometidos a un proceso intermedio de molienda y prensado isostático, se desarrolla una microestructura de $\mathrm{Al}_{2} \mathrm{TiO}_{5}$ conformada por granos porosos de $\mathrm{Al}_{2} \mathrm{TiO}_{5}$ del orden de $20 \mu \mathrm{m}$ (figura 5), los cuales son 
mucho menores que las celdas de $\mathrm{Al}_{2} \mathrm{TiO}_{5}(100 \mu \mathrm{m})$, formadas en los compactos tratados con un ciclo térmico simple. Debido al tratamiento intermedio de molienda, se rompen los aglomerados iniciales que conforman la microestructura de los compactos de $\mathrm{Al}_{2} \mathrm{TiO}_{5}$ sometidos a un tratamiento térmico simple y, se originan nuevas superficies de contacto en los compactos, dando lugar a la desaparición de partículas residuales de alúmina, de mayor tamaño $(\approx 5$ a $8 \mu \mathrm{m})$ y localizadas en borde de grano (figuras $4 \mathrm{c}, 4 \mathrm{~d}$ vs figura 5 ).

La creación de nuevas superficies durante la molienda favorece la densificación de los compactos, por lo que los materiales preparados utilizando el ciclo térmico en dos etapas (AT-temperatura -temperatura $_{2}$ ), presentan los valores mas altos de densidad, independientemente de la temperatura máxima utilizada (tabla II). Este hecho afecta a la efectividad final de la molienda.

El proceso de trituración de los compactos de $\mathrm{Al}_{2} \mathrm{TiO}_{5}$, seleccionados por su mayor grado de reacción, previo a la molienda de atrición, es más efectivo en los compactos con un tratamiento térmico en dos etapas, como se observó en las curvas para tiempos de molienda cero de la figura $6 \mathrm{a}\left(\mathrm{d}_{50} \approx 54 \mu \mathrm{m}\right.$ en AT- 1650 vs $d_{50} \approx 38 \mu \mathrm{m}$ en AT-1400-1600). Este hecho puede ser atribuido al tipo de microestructura que poseen los compactos seleccionados (figuras $4 \mathrm{~d}, 5 \mathrm{a}$ ): una matriz continua de $\mathrm{Al}_{2} \mathrm{TiO}_{5}$ con grandes grietas alargadas en los compactos AT-1650 vs celdas de $\mathrm{Al}_{2} \mathrm{TiO}_{5}$ del orden de $20 \mu \mathrm{m}$ en AT-1400-1600. En estos últimos compactos, el proceso de trituración sólo implicaría la separación de las celdas de $\mathrm{Al}_{2} \mathrm{TiO}_{5}$, siendo más difícil romper, la matriz continua de $\mathrm{Al}_{2} \mathrm{TiO}_{5}$ de los compactos AT- 1650 .

La efectividad de la molienda de atrición es mayor para los polvos de AT-1650 que para los polvos AT-1400-1600. Al aumentar el tiempo de molienda las distribuciones se desplazan hacia tamaños inferiores. A partir de una hora de molienda de atrición: los polvos AT-1650 presentan un $\mathrm{d}_{50} \approx 3 \mu \mathrm{m}$ hasta alcanzar en ocho horas de molienda un $\mathrm{d}_{50} \approx 0.5 \mu \mathrm{m}$ y, la molienda de los polvos AT-1400-1600 presenta un $\mathrm{d}_{50} \approx 6 \mu \mathrm{m}$ hasta alcanzar en ocho horas de molienda un $\mathrm{d}_{50} \approx 1.2 \mu \mathrm{m}$. A partir de ocho horas de molienda, para ambos materiales, la molienda de atrición deja de ser efectiva, no hay disminución de los tamaños promedios y las zonas centrales ( $80 \%$ en volumen) de las distribuciones no varían.

No sólo es importante considerar en las curvas de distribución de tamaños de partícula (figuras 6), donde esta centrada su distribución (80\% en volumen), los extremos de las curvas indican el comportamiento de las proporciones de tamaños de partículas de mayor tamaño ( $10 \%$ en volumen) y de menor tamaño ( $10 \%$ en volumen). Se ha observado en este estudio cómo, a partir de dos horas de molienda, en los polvos AT-14001600 las partículas con tamaños inferiores comienzan a aglomerarse, notándose a partir de estos tiempos de molienda, mayor inestabilidad en la molienda de los polvos de $\mathrm{Al}_{2} \mathrm{TiO}_{5}$ obtenidos a partir de los compactos AT-1400-1600.

De este estudio, se ha seleccionado como proceso optimo para la obtención de polvos de $\mathrm{Al}_{2} \mathrm{TiO}_{5}$ de alta pureza con $\mathrm{d}_{50} \approx 0.5 \mu \mathrm{m}$, la molienda de atrición de compactos de $\mathrm{Al}_{2} \mathrm{TiO}_{5^{\prime}}$ elaborador a partir de los compactos AT-V, con un tratamiento térmico a $1650^{\circ} \mathrm{C}$ y tres horas de permanencia a la temperatura máxima de tratamiento.

\section{CONCLUSIÓN}

Se ha analizado el efecto que tienen las condiciones de tratamiento térmico en el grado de reacción del $\mathrm{Al}_{2} \mathrm{TiO}_{5}$, el tamaño de las celdas de $\mathrm{Al}_{2} \mathrm{TiO}_{5}$ formadas y en la eficiencia de la posterior molienda de los materiales. Comprobándose que, a temperaturas cercanas a la temperatura de reacción (1350$1400^{\circ} \mathrm{C}$ ), la microestructura formada esta constituida por celdas porosas de $\mathrm{Al}_{2} \mathrm{TiO}_{5}$, de tamaño independiente de la temperatura de tratamiento y que poseen en su interior gran cantidad de partículas de $\mathrm{Al}_{2} \mathrm{O}_{3}$ y $\mathrm{TiO}_{2}$ sin reaccionar. El aumento de la temperatura $\left(1500-1650^{\circ} \mathrm{C}\right)$, supone grados de reacción superiores, hasta que la reacción es prácticamente completa, salvo la presencia de partículas aisladas de $\mathrm{Al}_{2} \mathrm{O}_{3}$. Por otra parte, para obtener polvos de $\mathrm{Al}_{2} \mathrm{TiO}_{5}$ de granulometria fina $\mathrm{y}$ homogénea, se ha comprobado que, los polvos de $\mathrm{Al}_{2} \mathrm{TiO}_{5}$ obtenidos a partir de un tratamiento térmico en una sola etapa, alcanzan en ocho horas de molienda un $\mathrm{d}_{50} \approx 0.5 \mu \mathrm{m}$, mientras que los polvos obtenidos a partir de un tratamiento térmico en dos etapas, alcanza en ocho horas de molienda un $\mathrm{d}_{50} \approx 1.2 \mu \mathrm{m}$. Estos resultados indican que, el tipo de microestructura de $\mathrm{Al}_{2} \mathrm{TiO}_{5}$ que se forma durante la reacción en estado sólido, afecta al posterior proceso de molienda de estos materiales, siendo más efectiva la molienda de los polvos de $\mathrm{Al}_{2} \mathrm{TiO}_{5}$ sometidos a un tratamiento térmico en una etapa.

\section{AGRADECIMIENTOS}

Los autores agradecen la ayuda económica recibida de la CYCYT a través del proyecto MAT 96-4048 y del Consejo Nacional de Investigaciones Científicas y Tecnológicas de Venezuela "CONICIT".

\section{BIBLIOGRAFÍA}

1 B. Freudenberg y A. Mocellin, "Aluminium Titanate Formation by Solid-State Reaction of Fine $\mathrm{Al}_{2} \mathrm{O}_{3}$ and $\mathrm{TiO}_{2}$ Powders," J. Am. Ceram. Soc., 70 [1] 33-38 (1987).

2 B. Freudenberg y A. Mocellin, "Aluminium Titanate Formation by Solid-State Reaction of Coarse $\mathrm{Al}_{2} \mathrm{O}_{3}$ and $\mathrm{TiO}_{2}$ Powders," J. Am. Ceram. Soc., 71 [1] 22-28 (1988).

3 W.D. Kingery, H.K. Bowen y D.R. Uhlmann. Introduction to Ceramics. Second Edition. A Wiley-Interscience Publication John Wiley \&Sons, pp.381-385 (1975).

4 H. A. J. Thomas and R. Stevens, "Microstructure Development During the Reaction Sintering of Alumina and Titania to Produce Aluminium Titanate". Brit. Cer. Proc. N ${ }^{\circ} 42$, Complex Microstructures, Ed.: R. Stevens and D. Taylor The Institute of Ceramics, Stoke-on-Trent, pp. 117-122, (1989).

5 V. Buscaglia, P. Nanni, G.Battilana, G. Aliprandi and C. Carry, "Reaction Sintering of Aluminium Titanate: I Effect of MgO Addition", J. Eur. Ceram. Soc., 13 411-417 (1994).

6 V. Buscaglia, P. Nanni, G.Battilana, G. Aliprandi and C. Carry, "Reaction Sintering of Aluminium Titanate: II Effect of Different Alumina Powders", J. Eur. Ceram. Soc., 13 419-426 (1994).

7 V. Buscaglia, M.A. Delfrate,P. Nanni, M.Leoni y C.Bottino: “Factors affecting microstructure evolution during reaction sintering of $\mathrm{Al}_{2} \mathrm{TiO}_{5}$ ceramics", $\mathrm{Adv}$. Sci. Technol., 3C., Edit. P.Vincenzini, 1867-1874(1995).

8 V. Buscaglia, M.A. Delfrate, M.Leoni y C.Bottino "The effect of $\mathrm{MgAl}_{2} \mathrm{O}_{4}$ on the formation kineticts of $\mathrm{Al}_{2} \mathrm{TiO}_{5}$ from $\mathrm{Al}_{2} \mathrm{O}_{3}$ and $\mathrm{TiO}_{2}$ fine powders", J. Matr. Sci., 31 1715-1724 (1996).

9 H. Wohlfromm, "Preparación por sinterización reactiva y estudio del comportamiento mecánico y tèrmico de materiales de titanato de aluminio", Tesis Doctoral, Universidad Autónoma de Madrid, 1991.

Recibido: 24-06-99

Aceptado: 18-11-99 